Article

\title{
Separation of Permethylated $O$-Glycans, Free Oligosaccharides, and Glycosphingolipid-Glycans Using Porous Graphitized Carbon (PGC) Column
}

\author{
Byeong Gwan Cho ${ }^{\circledR}$, Wenjing Peng and Yehia Mechref * \\ Department of Chemistry and Biochemistry, Texas Tech University, Lubbock, TX 79409, USA; \\ andrew.cho@ttu.edu (B.G.C.); wenjing.peng@ttu.edu (W.P.) \\ * Correspondence: yehia.mechref@ttu.edu
}

Received: 20 September 2020; Accepted: 21 October 2020; Published: 27 October 2020

\begin{abstract}
Glycosylation is one of the most common and complex post-translational modifications of proteins. However, there are other carbohydrates such as free oligosaccharides and glycosphingolipids-glycans that are associated with important biological and clinical roles. To analyze these molecules using liquid chromatography coupled with mass spectrometry (LC-MS), the permethylation approach was utilized. Although permethylation is a commonly utilized glycan derivatization technique, separation of permethylated glycans released from glycosphingolipid (GSL) by LC-MS has never been previously demonstrated. Here, a nanoflow porous graphitized carbon (PGC) column coupled with a high-resolution mass spectrometer was used to achieve isomeric separation of these permethylated glycans. We demonstrate the separation of free reducing end and reduced end $O$-glycans, free oligosaccharides derived from human milk, and GSL glycans derived from the MDA-MB-231BR cancer cell line using PGC-LC-MS.
\end{abstract}

Keywords: glycomics; LC-MS; PGC; glycosphingolipids; free oligosaccharides; O-glycans

\section{Introduction}

As a post-translational modification (PTM), glycosylation is one of the most complex and perhaps the most essential modifications of the protein [1]. In fact, its biological functions include immune cell trafficking [2], cell-to-cell adhesion [3] and signaling [4], protein stability [5], and more [6]. Due to the many important physiological roles that glycans play, their importance has been frequently highlighted by researchers from multiple disciplines. Most significantly, glycans' role in diseases such as cancer [7-12], neurodegenerative disorders [13,14], and diabetes [15,16] have been extensively researched. More recently, glycans have been associated with coronaviruses' spike glycoproteins, including that SARS-CoV-2 virion that causes COVID-19 [17].

Glycosylation is most frequently associated with protein glycosylation, although there are multifarious glycoconjugates, including glycolipids $[18,19]$. $N$-glycosylation and O-glycosylation of proteins are the two major glycoconjugates. $\mathrm{N}$-glycosylation involves glycans with a specific core structure being attached to the asparagine, with asparagine- $X$-serine/threonine amino acid sequence and $\mathrm{X}$ being any amino acid (except proline). O-glycosylation, on the other hand, occurs on the serine or threonine residue with no specific core structure, yielding a more complex structure [20]. Glycosphingolipids (GSL) are another class of glycoconjugates, wherein a glycan head group is attached to either a ceramide or sphingosine. These can be subclassified into groups such as gangliosides, globosides, and (neo)lactoseries, based on the core structure of their glycan heads. GSL, like glycoproteins, play important biological roles such as those related to immune cell functions [21]. Free oligosaccharides, perhaps the most interesting and abundant carbohydrates in milk, are also 
involved in the essential biological and clinical functions of the developing immune system in infants [22].

To perform analyses of these complex biomolecules, liquid chromatography coupled with mass spectrometry (LC-MS) is frequently employed to (1) separate convoluted isomeric glycans, (2) detect them with high mass accuracy and sensitivity, and (3) perform fragmentations with the goal of correct structural assignment. The analyses of glycans using LC-MS can differ based on the derivatization techniques utilized for each experiment [23]. For example, reduced native glycans can be analyzed using porous graphitized carbon (PGC) columns [24,25], while permethylated glycans are separated by C18 columns. This approach exploits the hydrophobicity of permethylated glycans [26] and therefore increases ionization efficiency, prompting higher sensitivity [27]. More recently, isomeric separation of permethylated N-glycans using PGC columns has been demonstrated by Zhou et al. to preserve higher sensitivity facilitated by permethylation while delivering isomeric separation [28].

We have reported the efficient isomeric separation of $N$-glycans previously [28-31]. Here, we demonstrate the separation of smaller glycans such as $O$-glycans, free oligosaccharides, and glycosphingolipid-glycans (GSL-glycans) using permethylation and PGC-LC-MS to resolve isomeric glycans at a high sensitivity. Glycans used in this study have been derived from a variety of biological samples such as bovine fetuin, k-casein, human milk, and the 231BR cancer cell line to demonstrate the applicability of the technique.

\section{Results}

\subsection{Separation of O-Glycans}

To examine the viability of $O$-glycan separation, $O$-glycans derived from standard glycoproteins were first assessed. Figure 1 shows the separation of permethylated $O$-glycans released from $\mathrm{k}$-casein, with insets depicting MS2 for each isomer. To reduce chromatographic bias, $\mathrm{O}$-glycans were multiplexed [32] during the permethylation with ${ }^{13} \mathrm{C}$-labeled methyl iodide, as shown in Figure 1a. Figure $1 \mathrm{~b}$ depicts permethylated $O$-glycans treated with an exoglycosidase, $\alpha 2-3$ neumeridase, and permethylated with ${ }^{12} \mathrm{C}$ methyl iodide. It is worth noting that the $O$-glycans illustrated here are free reducing end form, and therefore $\alpha$ and $\beta$ anomeric separation was observed. Interestingly, anomeric separation for GalNAc $\mathrm{Gal}_{1} \mathrm{NeuAc}_{1}$ was not observed, although GalNAc1(Gal1)-NeuAc1 displayed anomeric separation.

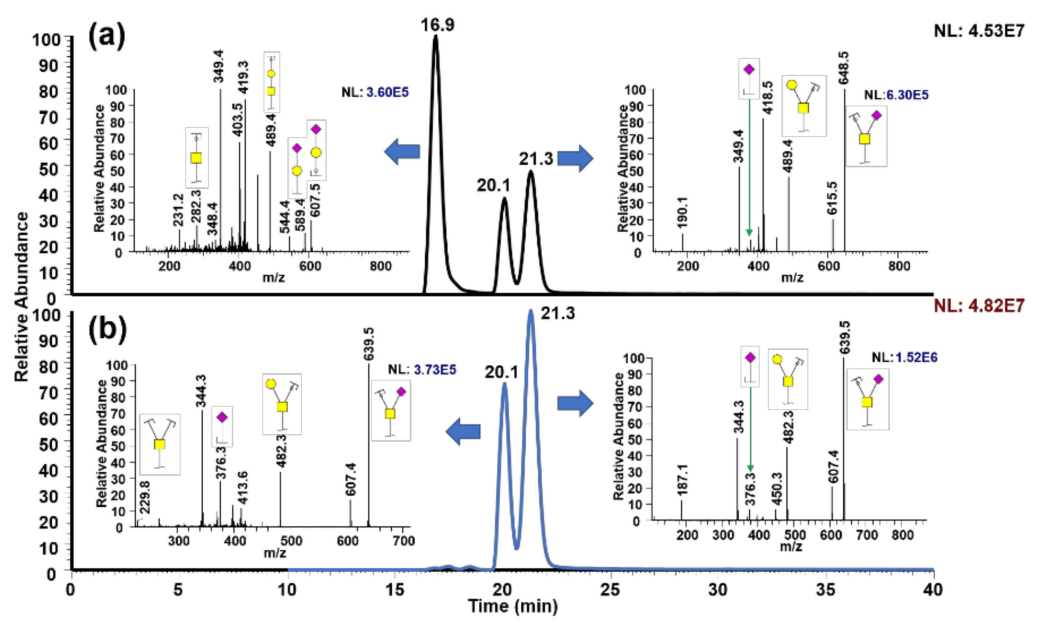

Figure 1. Isomeric separation of permethylated O-glycan isomers derived from k-casein using porous graphitized carbon-liquid chromatography coupled with mass spectrometry (PGC-LC-MS). (a) and (b) are examples of $O$-glycan isomers: the peak at $16.9 \mathrm{~min}$ represents GalNAc-Gal-NeuAc, and the peaks at 20.1 and 21.3 min represent anomers of GalNAc(NeuAc)-Gal. Insets are MS2 spectra of corresponding peaks. 
To assess whether anomeric separation would occur in other O-glycans, permethylated O-glycans with free reducing end derived from bovine fetuin were separated using PGC-LC-MS, as shown in Figure 2. GalNAc-Gal-NeuAc showed no anomeric separation; however, GalNAc(Gal-NeuAc)-GlcNAc-Gal-NeuAc exhibited anomeric separation. The MS2 spectra shown for each peak demonstrated that both peaks were the correct structural assignment.

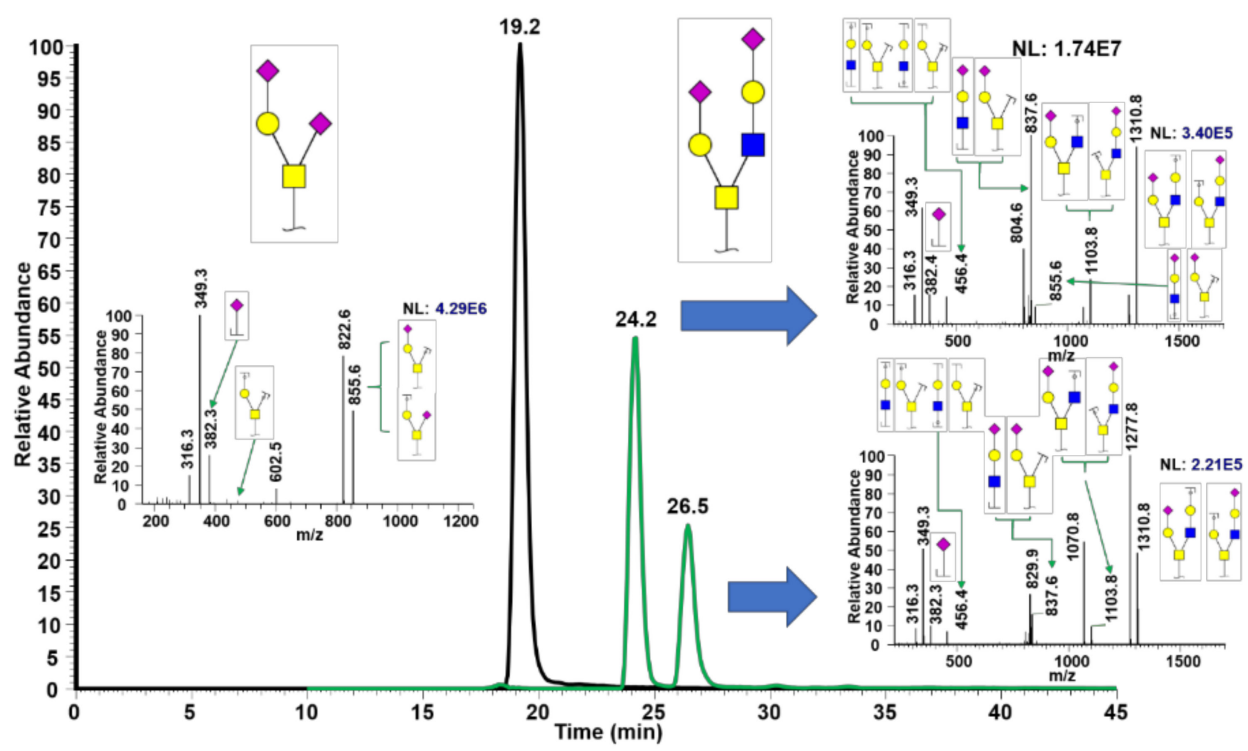

Figure 2. Chromatogram depicting two of the permethylated O-glycans derived from bovine fetuin using PGC-LC-MS. Insets are MS2 spectra for the corresponding peaks.

Expectedly, the determination of isomeric glycans with free reducing ends is more complicated due to unwanted anomeric separation, which results in increased chromatographic complexity. Therefore, reduced reducing end O-glycans were examined with similar experimental approaches to those shown in Figure 1. O-glycans derived from k-casein were permethylated with ${ }^{12} \mathrm{C}$ methyl iodide, and O-glycans with $\alpha 2-3$ neuraminidase treatment were permethylated with ${ }^{13} \mathrm{C}$, as shown in Figure 3, where the separation of two isomers is clearly demonstrated.

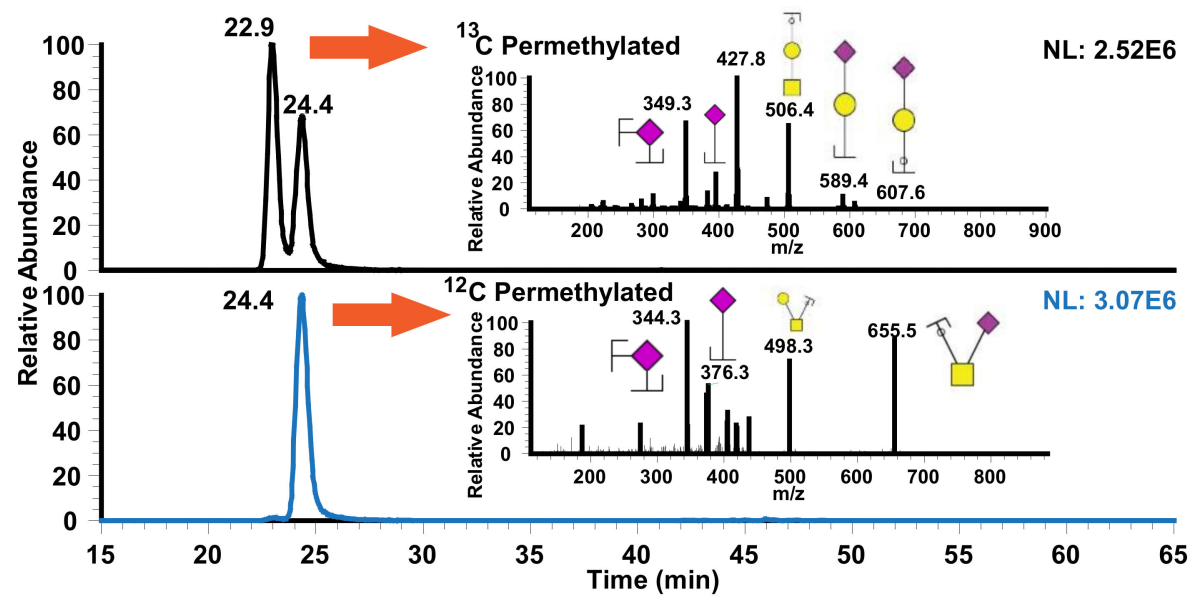

Figure 3. Separation of a reduced trisaccharide O-glycan derived from k-casein using PGC-LC-MS. Insets depict MS2 for the corresponding structures.

The effect of column temperature was also assessed, as shown in Figure 4. Permethylated and reduced O-glycans derived from bovine fetuin were analyzed using PGC-LC-MS at four different 
temperature settings: (a) $25^{\circ} \mathrm{C}$, (b) $45^{\circ} \mathrm{C}$, (c) $55^{\circ} \mathrm{C}$, and (d) $75^{\circ} \mathrm{C}$. Increasing the temperature had a significant effect on peak shape and resolution. As temperature increased, permethylated glycans eluted later, which was in agreement with the previous work by Zhou et al. [28] where permethylated $N$-glycan separation at high temperature was performed.

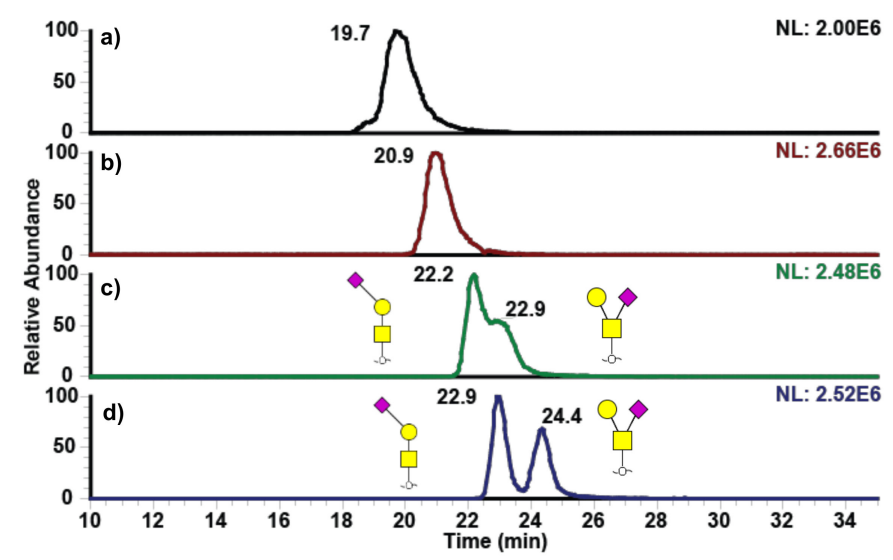

Figure 4. Effect of high temperature in separation of O-glycan. Panel (a) separation at $25^{\circ} \mathrm{C}$, (b) separation at $45^{\circ} \mathrm{C}$, (c) separation at $55^{\circ} \mathrm{C}$, and (d) separation at $75^{\circ} \mathrm{C}$.

Next, separation of $O$-glycans derived from proteins of human milk was performed using PGC-LC (Figure 5) to show the applicability of the method with biological samples. It is noteworthy that the chromatograms shown in Figure 5 represent free reducing end $O$-glycans. This approach was chosen due to increased sensitivity as a result of the enzymatic/chemical release of $O$-glycans demonstrated by Goetz et al. [33].

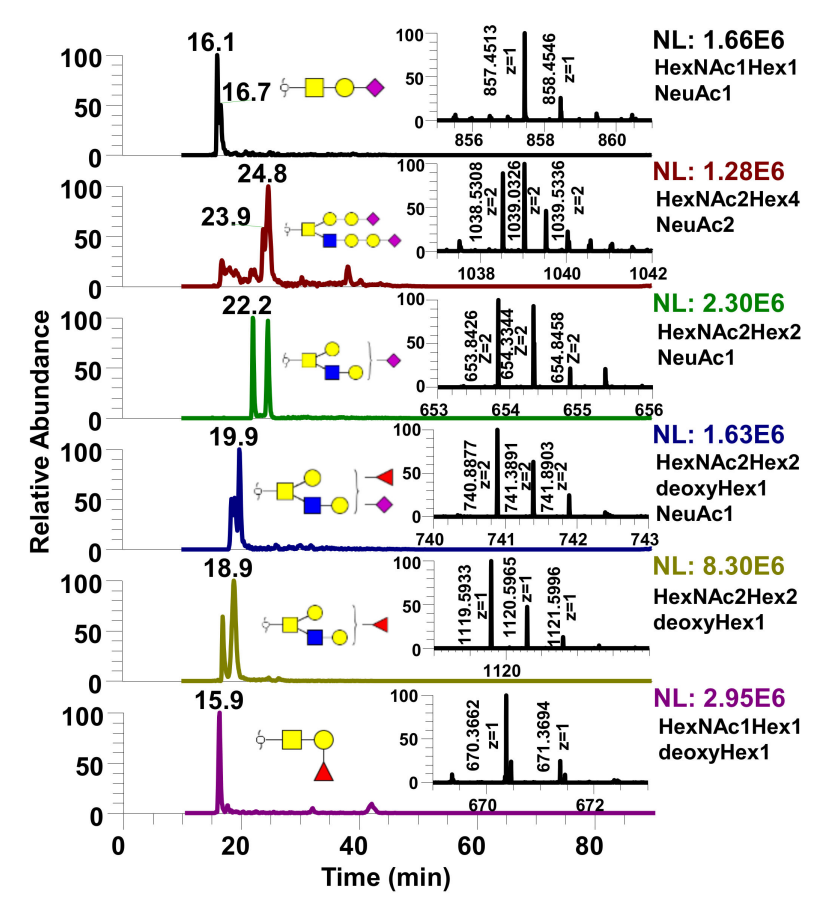

Figure 5. Extracted ion chromatograms (EIC) of various free reducing end O-glycans derived from human milk. Insets are mass spectrum of each O-glycan structure. 


\subsection{Separation of Free Oligosaccharides and Glycosphingolipid Glycans}

As previously mentioned, free oligosaccharides and GSL-glycans are other important glycoconjugates in biological and physiological systems. An analysis of free oligosaccharides from human milk is shown in Figure 6. Unlike O-glycans, these are free sugars and are not attached to any other bioconjugates, so no release step was needed. Therefore, the free oligosaccharides were reduced and then permethylated to avoid anomeric separation. Notably, separation of focusylated oligosaccharides was achieved using this technique.

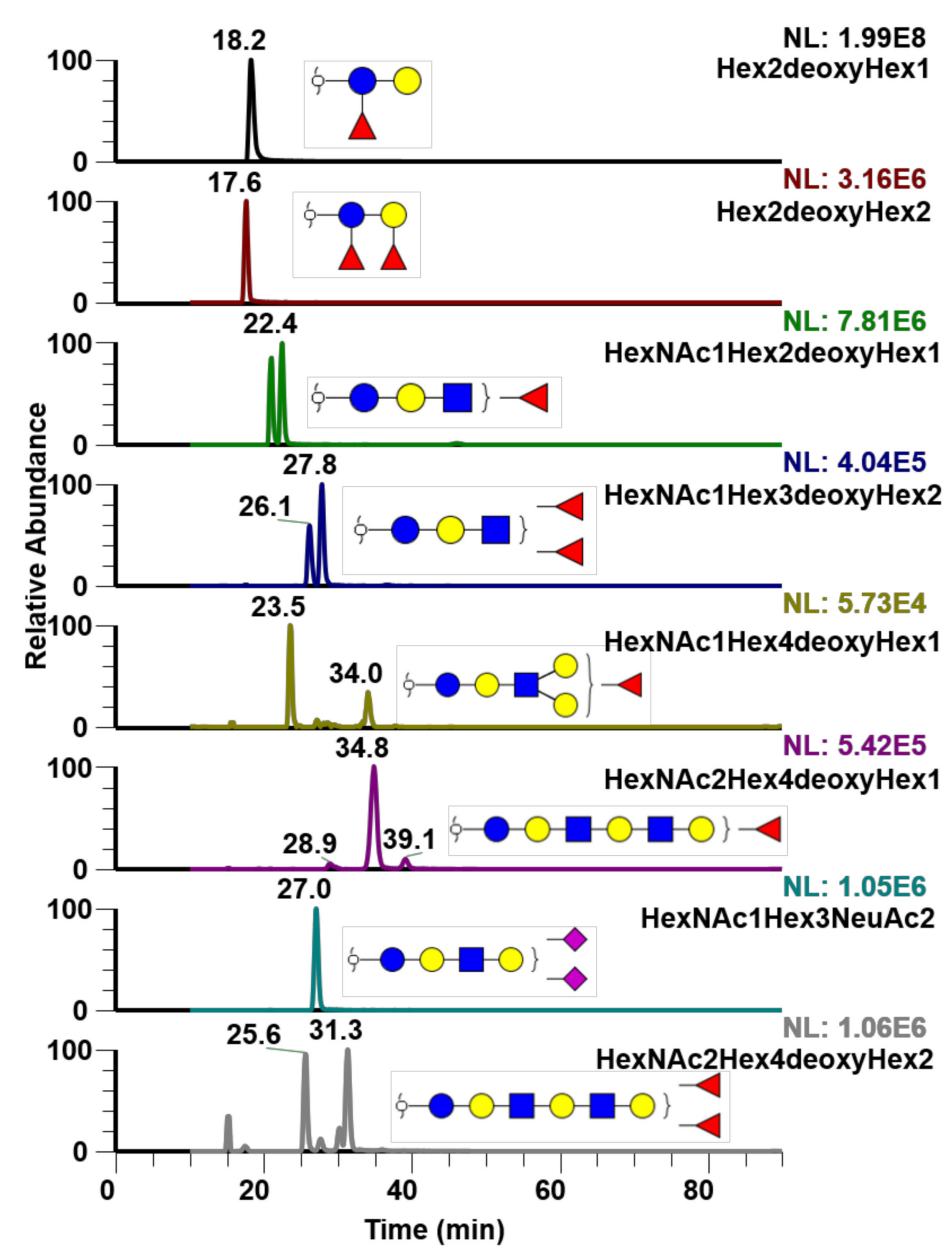

Figure 6. EIC (Extracted ion chromatogram) of free oligosaccharides extracted from human milk. Isomeric separation of some free oligosaccharides was achieved using PGC-LC-MS. MS2 spectra of the displayed structures were depicted in Figures S1-S7.

Furthermore, GSL-glycans were analyzed using PGC-LC-MS, as shown in Figure 7. In this case, GSL-glycans derived from breast cancer cell line 231BR were analyzed using PGC-LC-MS. Endoglycoceramidase I was utilized to release the glycans from GSL, which were then purified, reduced, and permethylated prior to analysis. The glycan head group from GSL was then analyzed. 

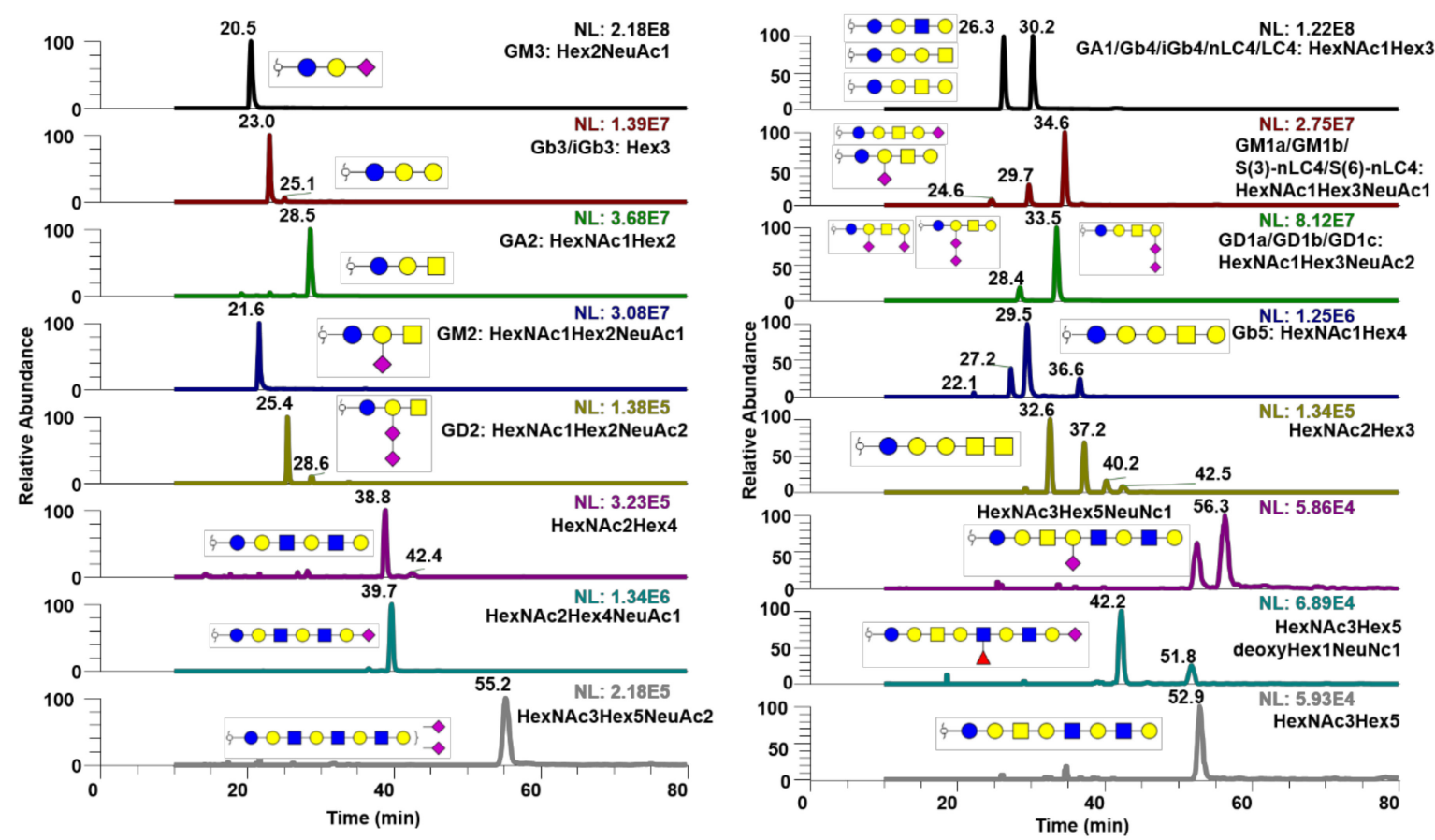

Figure 7. EIC of various glycosphingolipid (GSL) glycans derived from the 231BR cell line. Isomers of GSL-glycans are depicted with captions of their possible identity. MS2 spectra of the displayed structures were depicted in Figures S8-S16.

\section{Discussion}

In this work, we demonstrated isomeric separation of permethylated O-glycans, free oligosaccharides, and GSL-glycans using a PGC-LC technique that has been previously shown to separate permethylated $\mathrm{N}$-glycans [28-31]. Several separation techniques for $O$-glycans were used, including the utilization of ion mobility [34] and PGC-LC [34,35] for analysis of native O-glycans. Interestingly, in this study, anomeric as well as isomeric O-glycan separation was observed, as shown in Figure 1; Figure 2. Furthermore, GalNAc(NeuNc)-Gal and GalNAc(GlcNAc-Gal-NeuAc)Gal-NeuAc showed anomers being separated, while GalNAc-Gal-NeuAc and GalNAc(NeuAc)-Gal-NeuAc did not show anomers. To confirm this assertion, MS2 spectra, exoglycosidase, $\alpha 2-3$ neuraminidase, and multiplexing permethylation were employed (Figures 1 and 2). However, we note that glycan anomers are biologically irrelevant. The anomerization of alpha and beta anomers on the reducing ends of the glycan is a reversable process, once glycans are liberated from glycoproteins or other glycoconjugates. Not only are anomeric glycans irrelevant, they also interfere with the identification of glycan isomers by complicating chromatograms, making it impossible to pinpoint whether a given peak is an isomer or an anomer. Therefore, we examined permethylated reduced reducing end O-glycans derived from k-casein, as shown in Figure 3. Using the same technique applied in Figures 1 and 2, effective separation of sialic acid-linked $O$-glycan isomers was demonstrated. The peak at 22.9 min indicates GalNAc-Gal-NeuAc while the peak at 24.4 min indicates GalNAc(NeuAc)-Gal, as validated using both MS2 and $\alpha 2-3$ neuraminidase. It is important to mention the intensity differences between non-reduced $O$-glycans (Figure 1 ) and reduced $O$-glycans (Figure 3 ), where non-reduced $O$-glycans show more than 10 times the intensity of reduced $O$-glycans. This observation was in agreement with the previous study from Goetz et al. [33], where the intensity of $O$-glycans was 10 times higher compared to reduced and permethylated O-glycans, after using protease and pronase to digest the glycoprotein followed by permethylation to liberate and permethylate the O-glycans simultaneously.

While the analysis of permethylated $\mathrm{N}$ - and $\mathrm{O}$-glycans using PGC-LC-MS was initially performed by Cipollo et al. [36], separation of sialylated O- and N-glycans were not demonstrated. However, our work demonstrates the analysis of permethylated sialylated O-glycans at high temperatures. 
Using high temperature for the separation of permethylated N-glycans was previously demonstrated by Zhou et al. [28]. Utilizing the similar approach, the effect of PGC column temperature for separation of permethlyated O-glycan was investigated, as shown in Figure 4. Increased temperature drastically improved the peak shape and resolution of positional isomers, GalNAc-Gal-NeuAc and GalNAc(Gal)-NeuAc. This result revealed that permethylated O-glycans have a similar effect as permethylated N-glycans when separated on PGC column at high temperature.

Due to the increase in intensity, O-glycans were derived from human milk using an enzymatic/chemical method, as shown in Figure 5. As mentioned above, when both anomers and isomers were present in the sample, it was difficult to distinguish the two since some free reducing end O-glycans show anomeric separation while some do not. Therefore, researchers face a tradeoff between preserving sensitivity or maintaining simple chromatography for better identification of glycan isomers.

Next, free oligosaccharides from human milk were analyzed using PGC-LC-MS (Figure 6). It is worth noting that free oligosaccharides from human milk were the result of simply collecting the flow through a 10k molecular weight cut-off (MWCO) filter. Proteins collected with the filter were utilized to derive the O-glycans shown in Figure 5. Analysis of free oligosaccharides from human milk has been performed most recently by Porfirio et al. [37], and in previous research by Mank et al. [38], Xu et al. [39], and Dong et al. [40]. Mank et al. and Xu et al. selected a PGC column to analyze native reduced free oligosaccharides, while Porfirio et al. utilized a C18 reverse phase column to analyze permethylated free oligosaccharides without showing isomers of the free oligosaccharides. Dong et al. used both C18 and PGC columns to analyze the free oligosaccharides from different milk sources, however only one compositional glycan showing two isomers was exemplified. As shown in Figure 6, various isomers of each free oligosaccharide are depicted. It is important to note, however, that the oligosaccharides shown here are reduced reducing end oligosaccharides. Therefore, there are no anomers complicating the chromatograms.

Lastly, GSL-glycans derived from the 231BR cancer cell line were separated using PGC-LC, as shown in Figure 7. This is the first time that permethylated GSL-glycans have been analyzed using an LC-MS system, others have preferred 2-aminobenzamide (2-AB) labeling [41] or native [42] glycan analysis, although permethylation of intact glycosphingolipids [43] has been previously demonstrated. This work reveals that permethylated GSL-glycan isomers can be separated using PGC-LC while preserving the sensitivity that permethylation delivers to mass spectrometers. However, identification of these isomers can be tricky due to the lack of structural differences between some of the GSL-glycans. For example, there are four possible GSL-glycan isomers for HexNAc1Hex3. The only difference between neolacto 4 and lacto 4 is the beta linkage on the outer galactose. Nevertheless, PGC-LC allows separation of permethylated GSL-glycan isomers. For future work, it might be intriguing to investigate GSL-glycan profiles of different cancer cell lines or biological fluids from patients of various diseases, as well as to assign glucose units for these glycans, as shown by Gautam et al. [26]. The biggest pitfall of this technique may be the fact that the column used in this work is no longer produced by ThermoFisher. However, some researchers have already demonstrated that in-house packed PGC columns work just as well, as shown by Xiao et al. [44].

\section{Materials and Methods}

Bovine fetuin, k-casein, trifluoracetic acid, borane-ammonia complex, methyl iodide, methyl iodide $\left({ }^{13} \mathrm{C}\right)$, and sodium hydroxide beads were purchased from Sigma-Aldrich. High performance liquid chromatography (HPLC) water was obtained from Avantor. Methanol, acetonitrile, dimethyl sulfoxide (DMSO), and ammonium hydroxide were acquired from Fisher Scientific. Formic acid was from Tokyo Chemical Industry. 10k cut-off membrane filter units were purchased from MilliporeSigma. The C18 solid phase extraction cartridge and PGC column were from ThermoFisher (Thermo Fisher Scientific, Waltham, MA, USA). Activated charcoal spin columns and C8 spin columns were purchased from Harvard Apparatus. Endoglycocermidase I and $\alpha 2-3$ Neuraminidase $S$ were bought from 
New England Biolabs. Pronase was purchased from Roche. Cell line MDA-MB-231BR was a generous gift from Dr. Paul Lockman (West Virginia University, School of Pharmacy, Morgantown, WV, USA). The Dulbecco's Modified Eagle Medium (DMEM) and fetal bovine serum (FBS) for cell lines were purchased from ATCC. Trypsin-ethylenediaminetetraacetic acid (EDTA) 1 X (0.25\% Trypsin/2.21 mM EDTA) was purchased from Corning.

\subsection{O-Glycan Release}

Free reducing end O-glycans from bovine fetuin and k-casein were prepared by a chemical/enzymatic approach. $10 \mu \mathrm{g}$ of each glycoprotein was subjected to pronase digestion (Protein:Enzyme $=10: 1$ ) by incubating at $55^{\circ} \mathrm{C}$ for $48 \mathrm{~h}$. Then, the samples were dried and subjected to solid phase permethylation. Dried pronase digested samples were resuspended in $30 \mu \mathrm{L}$ of DMSO, $1.2 \mu \mathrm{L}$ of water, and $20 \mu \mathrm{L}$ iodomethane (either ${ }^{12} \mathrm{C}$ or ${ }^{13} \mathrm{C}$, depending on the experiment). Resuspended samples were then added to the sodium hydroxide bead-packed micro spin column and incubated for $25 \mathrm{~min}$ at room temperature. An additional $20 \mu \mathrm{L}$ of methyl iodide was added, then incubated for another $15 \mathrm{~min}$ at room temperature. After the incubation, spin columns were centrifuged at $1.8 \mathrm{k}$ $\mathrm{g}$ for $2 \mathrm{~min}$ at room temperature. Next, $50 \mu \mathrm{L}$ of acetonitrile was added to each spin column then centrifuged again at the same speed for $2 \mathrm{~min}$. Collected permethylated glycans were dried in the SpeedVac prior to analysis.

Reduced reducing end O-glycans were prepared according to the protocol by Huang et al. [45]. $10 \mu \mathrm{g}$ of glycoproteins were resuspended in $5 \mathrm{mg} / \mathrm{mL}$ borane-ammonia complex in ammonium hydroxide and incubated for $18 \mathrm{~h}$ at $45^{\circ} \mathrm{C}$. After incubation, the samples were dried in the SpeedVac and methanol was added to discard the borane. Finally, the samples were permethylated as described above.

\section{2. $\alpha 2$-3. Neuraminidase S Digestion}

For the samples with $\alpha 2-3$ neuraminidase S digestion, protocols from New England Biolabs were followed exactly. $5 \mu \mathrm{g}$ of glycoproteins were resuspended in $45 \mu \mathrm{L}$ of water, $5 \mu \mathrm{L}$ of Glycobuffer1, and $5 \mu \mathrm{L}$ of $\alpha 2-3$ neuraminidase $S$ and incubated for $1 \mathrm{~h}$ at $37^{\circ} \mathrm{C}$. Depending on the experiment, free reducing end $\mathrm{O}$-glycan or reduced reducing end $\mathrm{O}$-glycan procedures were followed as described above.

\subsection{Preparation of O-Glycans and Free Oligosaccharides from Human Milk}

The human milk sample was filtered through a 10k MWCO filter (MiliporeSigma, Burlington, MA, USA). The flow through was collected for free oligosaccharide analysis, and the proteins collected in the filter were used for O-glycan analysis according to the procedures described above. Free oligosaccharides were reduced by the addition of $10 \mathrm{mg} / \mathrm{mL}$ borane-ammonia complex with incubation for $1 \mathrm{~h}$ at $60{ }^{\circ} \mathrm{C}$. Methanol washes were performed until no white residues remained, and samples were permethylated as described above.

\subsection{Cell Line Cultivation}

Cell line MDA-MB-231BR (231BR) was stored in liquid nitrogen. One tube (1 mL) of stored 231BR was taken out, thawed in a $37^{\circ} \mathrm{C}$ water bath, and transferred to a $75 \mathrm{~cm}^{2}$ flask. Then, $11 \mathrm{~mL}$ of DMEM (with 20\% FBS and 2\% penicillin-streptomycin solution) was added to the flask. The cells were then incubated at $37{ }^{\circ} \mathrm{C}$ with $5 \% \mathrm{CO}_{2}$ for four days. During incubation, cells were fed every two days. After reaching an $80 \%$ cell confluence, cells were washed with $10 \mathrm{~mL}$ phosphate buffer saline (PBS). Next, cells were detached by adding $2.2 \mathrm{~mL}$ of trypsin-EDTA solution and incubating for $5 \mathrm{~min}$ at $37^{\circ} \mathrm{C}$. After incubation, $5 \mathrm{~mL}$ of fresh medium was added and mixed to neutralize the trypsin, followed by inoculating $1 \mathrm{~mL}$ to a $175 \mathrm{~cm}^{2}$ flask (three $175 \mathrm{~cm}^{2}$ flasks were prepared as three triplicates). Then, $24 \mathrm{~mL}$ of complete DMEM was added to the flask, and cells were incubated at $37{ }^{\circ} \mathrm{C}, 5 \% \mathrm{CO}_{2}$ for 5 days until an $80 \%$ cell confluence was reached. Cells were then washed with $20 \mathrm{~mL}$ PBS and detached with $5 \mathrm{~mL}$ trypsin-EDTA solution, as described above. After detachment, $10 \mathrm{~mL}$ of fresh medium was added and mixed. The cell culture was transferred to a $15 \mathrm{~mL}$ tube and centrifuged at $500 \times g$ for 
$5 \mathrm{~min}$ at room temperature. The cell pellet was washed two times with PBS to remove the remaining medium, and then stored at $-20^{\circ} \mathrm{C}$ for further analysis.

\subsection{GSL Extraction from 231BR Cell Line}

Cell lysate was subjected to lipid extraction using methanol and chloroform adopted from Albrecht et al. [41]. $100 \mu \mathrm{L}$ of chloroform and $200 \mu \mathrm{L}$ of methanol was added to $75 \mu \mathrm{L}$ of cell lysate, vortexed and sonicated for $5 \mathrm{~min}$, then centrifuged at $14 \mathrm{~kg}$ for $2 \mathrm{~min}$. Supernatant was collected and a mixture of chloroform and methanol (1:1) was added to the pallet, vortexed, sonicated, and centrifuged. The supernatant was pooled and the same steps were repeated with a mixture of chloroform and methanol (2:1). Pooled supernatant was dried in the SpeedVac, then a C8 spin column purification of glycosphingolipids was performed according to Benktander et al. [46]. Dried lipids were resuspended in a methanol and water mixture (3:2). C8 spin columns were washed with methanol three times, conditioned with a methanol and water mixture (3:2) twice, then the resuspended lipid sample was added to the spin column and centrifuged. Eluate was added back to the column and centrifuged, then the columns were washed with a methanol and water mixture (3:2) three times. Glycosphingolipids were eluted by applying methanol twice and drying in the SpeedVac.

\subsection{GSL-Glycan Preparation}

Dried GSL were resuspended in $14 \mu \mathrm{L}$ of water and $4 \mu \mathrm{L}$ of endoglycoceraminidase I (EGCase I) Buffer. $2 \mu \mathrm{L}$ of endoglycoceramidase I was added to the sample and incubated at $37^{\circ} \mathrm{C}$ for $18 \mathrm{~h}$. After the incubation, the samples were dried in the SpeedVac and resuspended in 5\% acetic acid (aq) for C18 purification. The $\mathrm{C} 18$ cartridges $(1 \mathrm{~mL})$ were washed with methanol three times and conditioned with $5 \%$ acetic acid (aq) three times. Samples were applied to the cartridges and flow through was collected. $5 \%$ acetic acid (aq) was applied three more times, pooled into the flow-through vial, and dried. Dried GSL-glycans were resuspended in $0.1 \%$ trifluoroacetic acid (TFA) in $85 \%$ acetonitrile and $15 \%$ water to perform solid phase extraction using activated charcoal spin columns. Spin columns were washed with $0.1 \%$ TFA in $85 \%$ acetonitrile and $15 \%$ water three times, then conditioned with $0.1 \%$ TFA in $5 \%$ acetonitrile and $95 \%$ water three times. Samples were applied to the columns and centrifuged, then eluants were reapplied to the column. This step was repeated twice. Next, the columns were washed with $0.1 \%$ TFA in $5 \%$ acetonitrile and $95 \%$ water twice before elution of glycans with $0.1 \%$ TFA, $50 \%$ acetonitrile, and 50\% water twice. Purified GSL-glycans were reduced as described in Section 4.3 and permethylated as described in Section 4.1.

\subsection{PGC-LC-MS}

Online purification of permethylated glycans was performed using a PepMap C18 Trapping Column $(0.075 \mathrm{~mm} \times 2 \mathrm{~cm}, 3 \mu \mathrm{m})$ which was equipped on an UltiMate 3000 RSLCNano system (Thermo Fisher Scientific, Waltham, MA, USA) connected to an LTQ Orbitrap Velos (Thermo Fisher Scientific, Waltham, MA, USA). The analytical column was the Thermo Scientific PGC (Thermo Fisher Scientific, Waltham, MA, USA) Hypercarb $(0.075 \mathrm{~mm} \times 10 \mathrm{~cm}, 5 \mu \mathrm{m})$. Flow rate was set at $600 \mathrm{~nL} / \mathrm{min}$ with temperature set at $75^{\circ} \mathrm{C}$. Mobile phase A was $0.1 \%$ formic acid in water, and mobile phase $\mathrm{B}$ was $0.1 \%$ formic acid in acetonitrile. The gradient was kept at $20 \% \mathrm{~B}$ for the first $10 \mathrm{~min}$ while online purification occurred. Then, the valve was switched to load purified permethylated glycans onto the analytical column at $10 \mathrm{~min}$. B was set to $60 \%$ at $25 \mathrm{~min}$ then increased to $95 \%$ at $46 \mathrm{~min}$ and kept for $38 \mathrm{~min}$. B was decreased to $20 \%$ for re-equilibration for $16 \mathrm{~min}$. The LTQ Orbitrap Velos was set at $1.6 \mathrm{kV}$, positive mode, with full MS performing at 100,000 resolution and scan range from 400 to $2000 \mathrm{~m} / \mathrm{z}$. Data-dependent acquisition was performed with collision-induced dissociation (CID) settings set to 5000 minimum signal required, 30 normalized collision energy, and 0.25 activation $Q$, and the top 6 peaks were subjected to fragmentation. 


\subsection{Data Processing}

Raw files were processed manually using Xcalibur 4.2 (Thermo Fisher Scientific, Waltham, MA, USA). Glycans were annotated with the assistance of Glycoworkbench 2. The table of contents was made with BioRender.com.

\section{Conclusions}

In this work, we demonstrated for the first time the use of PGC-LC-MS at high temperature to separate permethylated O-glycans, free oligosaccharides, and released GSL-glycans from cell lines. Our work showed the capability of PGC column for separating permethylated O-glycans, free oligosaccharides, as well as GSL-glycans. This work also investigated anomeric and isomeric separation of $O$-glycans. This method could be used to analyze isomeric $O$-glycans on antibody-based therapeutics but also could be utilized to examine the role of $O$-glycans in cancer and other diseases. The use of PGC-LC-MS for the analysis of permethylated free oligosaccharide and GSL-glycan isomers facilitated extensive isomeric separation as well as enhanced sensitivity allowed by enhanced ionization via permethylation. This technique can be adopted to further investigate GSL-glycan composition and their isomers derived from other cancer cell lines to examine potential glycolipid-based cancer biomarkers with the emphasis on the glycan head group.

Supplementary Materials: The supplementary materials are available online at http://www.mdpi.com/2218-1989/ 10/11/433/s1.

Author Contributions: Conceptualization, B.G.C. and Y.M.; methodology, B.G.C.; investigation, B.G.C.; resources, B.G.C. and W.P.; writing-original draft preparation, B.G.C.; writing-review and editing, B.G.C., W.P. and Y.M.; visualization, B.G.C.; supervision, Y.M.; project administration, B.G.C. and Y.M.; funding acquisition, Y.M. All authors have read and agreed to the published version of the manuscript.

Funding: This research was funded by National Institutes of Health (NIH), 1R01GM112490-04, 1R01GM130091-01, and 1U01CA225753-01.

Conflicts of Interest: The authors declare no conflict of interest.

\section{References}

1. Hart, G.W.; Copeland, R.J. Glycomics Hits the Big Time. Cell 2010, 143, 672-676. [CrossRef] [PubMed]

2. Sperandio, M.; Gleissner, C.A.; Ley, K. Glycosylation in immune cell trafficking. Immunol. Rev. 2009, 230, 97-113. [CrossRef] [PubMed]

3. Moriguchi, T.; Yu, L.; Schachter, H.; Wells, L.; Campbell, K.O. 1 Novel O-mannosyl phosphorylation of alpha-dystroglycan is required for laminin binding: Implications for congenital muscular dystrophy. Neuromuscul. Disord. 2010, 20, 597. [CrossRef]

4. De Vreede, G.; Morrison, H.A.; Houser, A.M.; Boileau, R.M.; Andersen, D.S.; Colombani, J.; Bilder, D. A Drosophila Tumor Suppressor Gene Prevents Tonic TNF Signaling through Receptor N-Glycosylation. Dev. Cell 2018, 45, 595-605. [CrossRef]

5. Solá, R.J.; Griebenow, K. Effects of glycosylation on the stability of protein pharmaceuticals. J. Pharm. Sci. 2009, 98, 1223-1245. [CrossRef] [PubMed]

6. Reily, C.; Stewart, T.J.; Renfrow, M.B.; Novak, J. Glycosylation in health and disease. Nat. Rev. Nephrol. 2019, 15, 346-366. [CrossRef]

7. Mechref, Y.; Hu, Y.; Garcia, A.; Hussein, A. Identifying cancer biomarkers by mass spectrometry-based glycomics. Electrophoresis 2012, 33, 1755-1767. [CrossRef]

8. Kailemia, M.J.; Park, D.; Lebrilla, C.B. Glycans and glycoproteins as specific biomarkers for cancer. Anal. Bioanal. Chem. 2016, 409, 395-410. [CrossRef]

9. Guo, H.; Abbott, K.L. Functional Impact of Tumor-Specific N-Linked Glycan Changes in Breast and Ovarian Cancers. Adv. Cancer Res. 2015, 126, 281-303.

10. Pan, S.; Brentnall, T.A.; Chen, R. Glycoproteins and glycoproteomics in pancreatic cancer. World J. Gastroenterol. 2016, 22, 9288-9299. [CrossRef] 
11. Mehta, A.; Herrera, H.; Block, T.M. Glycosylation and Liver Cancer. Adv. Cancer Res. 2015, 126, $257-279$. [PubMed]

12. Wooding, K.M.; Peng, W.; Mechref, Y. Characterization of Pharmaceutical IgG and Biosimilars Using Miniaturized Platforms and LC-MS/MS. Curr. Pharm. Biotechnol. 2016, 17, 788-801. [CrossRef]

13. Kizuka, Y.; Kitazume, S.; Taniguchi, N. N-glycan and Alzheimer's disease. Biochim. Biophys. Acta Gen. Subj. 2017, 1861, 2447-2454. [CrossRef] [PubMed]

14. Schedin-Weiss, S.; Winblad, B.; Tjernberg, L.O. The role of protein glycosylation in Alzheimer disease. FEBS J. 2013, 281, 46-62. [CrossRef] [PubMed]

15. Everest-Dass, A.V.; Moh, E.S.X.; Ashwood, C.; Shathili, A.M.M.; Packer, N.H. Human disease glycomics: Technology advances enabling protein glycosylation analysis-Part 2. Expert Rev. Proteom. 2018, 15, 341-352. [CrossRef] [PubMed]

16. Bermingham, M.L.; Colombo, M.; McGurnaghan, S.J.; Blackbourn, L.A.; Vučković, F.; Baković, M.P.; Trbojević-Akmačić, I.; Lauc, G.; Agakov, F.; Agakova, A.S.; et al. N-Glycan Profile and Kidney Disease in Type 1 Diabetes. Diabetes Care 2017, 41, 79-87. [CrossRef]

17. Walls, A.C.; Park, Y.-J.; Tortorici, M.A.; Wall, A.; McGuire, A.T.; Veesler, D. Structure, Function, and Antigenicity of the SARS-CoV-2 Spike Glycoprotein. Cell 2020, 181, 281-292. [CrossRef]

18. Varki, A.; Gagneux, P. Biological Functions of Glycans. In Essentials of Glycobiology, 1st ed.; Cold Spring Harbor: New York, NY, USA, 2015; pp. 77-88.

19. Cummings, R.D.; Pierce, J.M. The Challenge and Promise of Glycomics. Chem. Biol. 2014, 21, 1-15. [CrossRef]

20. Colley, K.J.; Varki, A.; Kinoshita, T. Cellular Organization of Glycosylation. In Essentials of Glycobiology; Cold Spring Harbor: New York, NY, USA, 2015; pp. 41-49.

21. Zhang, T.; De Waard, A.A.; Wuhrer, M.; Spaapen, R.M. The Role of Glycosphingolipids in Immune Cell Functions. Front. Immunol. 2019, 10, 90. [CrossRef]

22. Ayechu-Muruzabal, V.; Van Stigt, A.H.; Mank, M.; Willemsen, L.E.M.; Stahl, B.; Garssen, J.; Land, B.V. Diversity of Human Milk Oligosaccharides and Effects on Early Life Immune Development. Front. Pediatr. 2018, 6, 239. [CrossRef]

23. Dong, X.; Huang, Y.; Cho, B.G.; Zhong, J.; Gautam, S.; Peng, W.; Williamson, S.D.; Banazadeh, A.; Torres-Ulloa, K.Y.; Mechref, Y. Advances in mass spectrometry-based glycomics. Electrophoresis 2018, 39, 3063-3081. [CrossRef]

24. Zhang, T.; Madunic, K.; Holst, S.; Zhang, J.; Jin, C.; Dijke, P.T.; Karlsson, N.G.; Stavenhagen, K.; Wuhrer, M. Development of a 96-well plate sample preparation method for integrated N- and O-glycomics using porous graphitized carbon liquid chromatography-mass spectrometry. Mol. Omics. 2020, 16, 355-363. [CrossRef] [PubMed]

25. Ashwood, C.; Pratt, B.; MacLean, B.X.; Gundry, R.L.; Packer, N.H. Standardization of PGC-LC-MS-based glycomics for sample specific glycotyping. Analyst 2019, 144, 3601-3612. [CrossRef]

26. Gautam, S.; Peng, W.; Cho, B.G.; Huang, Y.; Banazadaeh, A.; Yu, A.; Dong, X.; Mechref, Y. Glucose unit index (GUI) of permethylated glycans for effective identification of glycans and glycan isomers. Analyst 2020, 145, 6656-6667. [CrossRef] [PubMed]

27. Zhou, S.; Veillon, L.; Dong, X.; Huang, Y.; Mechref, Y. Direct comparison of derivatization strategies for LC-MS/MS analysis of N-glycans. Analyst 2017, 142, 4446-4455. [CrossRef] [PubMed]

28. Zhou, S.; Huang, Y.; Dong, X.; Peng, W.; Veillon, L.; Kitagawa, D.A.S.; Aquino, A.J.A.; Mechref, Y. Isomeric Separation of Permethylated Glycans by Porous Graphitic Carbon (PGC)-LC-MS/MS at High Temperatures. Anal. Chem. 2017, 89, 6590-6597. [CrossRef]

29. Huang, Y.; Zhou, S.; Zhu, J.; Lubman, D.M.; Mechref, Y. LC-MS/MS isomeric profiling of permethylated N-glycans derived from serum haptoglobin of hepatocellular carcinoma (HCC) and cirrhotic patients. Electrophoresis 2017, 38, 2160-2167. [CrossRef]

30. Zhou, S.; Dong, X.; Veillon, L.; Huang, Y.; Mechref, Y. LC-MS/MS analysis of permethylated N-glycans facilitating isomeric characterization. Anal. Bioanal. Chem. 2016, 409, 453-466. [CrossRef]

31. Peng, W.; Goli, M.; Mirzaei, P.; Mechref, Y. Revealing the Biological Attributes of N-Glycan Isomers in Breast Cancer Brain Metastasis Using Porous Graphitic Carbon (PGC) Liquid Chromatography-Tandem Mass Spectrometry (LC-MS/MS). J. Proteome Res. 2019, 18, 3731-3740. [CrossRef] 
32. Dong, X.; Peng, W.; Yu, C.-Y.; Zhou, S.; Donohoo, K.B.; Tang, H.; Mechref, Y. 8-plex LC-MS/MS Analysis of Permethylated N-Glycans Achieved by Using Stable Isotopic Iodomethane. Anal. Chem. 2019, 91, 11794-11802. [CrossRef]

33. Goetz, J.A.; Novotny, M.V.; Mechref, Y. Enzymatic/Chemical Release of O-Glycans Allowing MS Analysis at High Sensitivity. Anal. Chem. 2009, 81, 9546-9552. [CrossRef]

34. Jin, C.; Harvey, D.J.; Struwe, W.B.; Karlsson, N.G. Separation of Isomeric O-Glycans by Ion Mobility and Liquid Chromatography-Mass Spectrometry. Anal. Chem. 2019, 91, 10604-10613. [CrossRef]

35. Xu, G.; Goonatilleke, E.; Wongkham, S.; Lebrilla, C.B. Deep Structural Analysis and Quantitation of O-Linked Glycans on Cell Membrane Reveal High Abundances and Distinct Glycomic Profiles Associated with Cell Type and Stages of Differentiation. Anal. Chem. 2020, 92, 3758-3768. [CrossRef] [PubMed]

36. Costello, C.E.; Contado-Miller, J.M.; Cipollo, J.F. A glycomics platform for the analysis of permethylated oligosaccharide alditols. J. Am. Soc. Mass Spectrom. 2007, 18, 1799-1812. [CrossRef]

37. Porfirio, S.; Archer-Hartmann, S.; Moreau, G.B.; Ramakrishnan, G.; Haque, R.; Kirkpatrick, B.D.; Petri, W.A.; Azadi, P. New strategies for profiling and characterization of human milk oligosaccharides. Glycobiology 2020, 30, 774-786. [CrossRef] [PubMed]

38. Mank, M.; Welsch, P.; Heck, A.J.; Stahl, B. Label-free targeted LC-ESI-MS2 analysis of human milk oligosaccharides (HMOS) and related human milk groups with enhanced structural selectivity. Anal. Bioanal. Chem. 2018, 411, 231-250. [CrossRef] [PubMed]

39. Xu, G.; Davis, J.C.; Goonatilleke, E.; Smilowitz, J.T.; German, J.B.; Lebrilla, C.B. Absolute Quantitation of Human Milk Oligosaccharides Reveals Phenotypic Variations during Lactation. J. Nutr. 2016, 147, 117-124. [CrossRef]

40. Dong, X.; Zhou, S.; Mechref, Y. LC-MS/MS analysis of permethylated free oligosaccharides and N-glycans derived from human, bovine, and goat milk samples. Electrophoresis 2016, 37, 1532-1548. [CrossRef] [PubMed]

41. Albrecht, S.; Vainauskas, S.; Stöckmann, H.; McManus, C.; Taron, C.H.; Rudd, P.M.; Stöckmann, H. Comprehensive Profiling of Glycosphingolipid Glycans Using a Novel Broad Specificity Endoglycoceramidase in a High-Throughput Workflow. Anal. Chem. 2016, 88, 4795-4802. [CrossRef]

42. Li, Q.; Xie, Y.; Wong, M.; Barboza, M.; Lebrilla, C.B. Comprehensive structural glycomic characterization of the glycocalyxes of cells and tissues. Nat. Protoc. 2020, 15, 2668-2704. [CrossRef]

43. Barrientos, R.C.; Zhang, Q. Differential Isotope Labeling by Permethylation and Reversed-Phase Liquid Chromatography-Mass Spectrometry for Relative Quantification of Intact Neutral Glycolipids in Mammalian Cells. Anal. Chem. 2019, 91, 9673-9681. [CrossRef] [PubMed]

44. Xiao, K.; Wang, Y.; Shen, Y.; Han, Y.; Tian, Z. Large-scale identification and visualization of N-glycans with primary structures using GlySeeker. Rapid Commun. Mass Spectrom. 2017, 32, 142-148. [CrossRef]

45. Huang, Y.; Konse, T.; Mechref, Y.; Novotny, M.V. Matrix-assisted laser desorption/ionization mass spectrometry compatible beta-elimination of O-linked oligosaccharides. Rapid Commun. Mass Spectrom. 2002, 16, 1199-1204. [CrossRef] [PubMed]

46. Benktander, J.D.; Gizaw, S.T.; Gaunitz, S.; Novotny, M.V. Analytical Scheme Leading to Integrated High-Sensitivity Profiling of Glycosphingolipids Together with N- and O-Glycans from One Sample. J. Am. Soc. Mass Spectrom. 2018, 29, 1125-1137. [CrossRef] [PubMed]

Publisher's Note: MDPI stays neutral with regard to jurisdictional claims in published maps and institutional affiliations.

(C) 2020 by the authors. Licensee MDPI, Basel, Switzerland. This article is an open access article distributed under the terms and conditions of the Creative Commons Attribution (CC BY) license (http://creativecommons.org/licenses/by/4.0/). 